\title{
Heat-moulded versus custom-made mandibular advancement devices for obstructive sleep apnoea: a randomised non-inferiority trial
}

\author{
Jean Louis Pépin, ${ }^{\oplus 1,2}$ Nathalie Raymond, ${ }^{3}$ Olivier Lacaze, ${ }^{4}$ Nathalie Aisenberg, ${ }^{5}$ \\ Jérôme Forcioli, ${ }^{6}$ Eric Bonte, ${ }^{7}$ Arnaud Bourdin, ${ }^{8}$ Sandrine Launois, ${ }^{9,10}$ \\ Renaud Tamisier, ${ }^{\oplus, 10}$ Nicolas Molinari ${ }^{11}$
}

For numbered affiliations see end of article.

\section{Correspondence to} Professor Jean Louis Pépin Universite Grenoble Alpes, Saint-Martin-d'Heres, France jpepin@chu-grenoble.fr

JLP and NR contributed equally.

Received 15 October 2018 Revised 7 March 2019 Accepted 18 March 2019 Published Online First 3 May 2019

\section{Linked}

- http://dx.doi.org/10.1136/ thoraxjnl-2019-213366

\section{Check for updates}

(C) Author(s) (or their employer(s)) 2019. No commercial re-use. See rights and permissions. Published by BMJ.

To cite: Pépin $J$,

Raymond N, Lacaze 0, et al.

Thorax 2019:74:667-674.

\section{ABSTRACT}

Rationale Custom-made mandibular advancement devices (MADs) are reported as providing higher efficacy rates compared with thermoplastic heat-moulded MADs but at the price of higher costs and treatment delays. Objective To determine whether a thermoplastic heat-moulded titratable MAD (ONIRIS; ONIRIS SAS, Rueil Malmaison, France) is non-inferior to a custommade acrylic titratable MAD (TALI; ONIRIS SAS, Rueil Malmaison, France) for obstructive sleep apnoea (OSA). Methods We conducted a multicentre, open, randomised controlled trial of patients with OSA refusing or not tolerating continuous positive airway pressure (CPAP). Participants were randomly assigned to a thermoplastic heat-moulded titratable device or a custom-made acrylic device for 2 months with stratification by centre and OSA severity. The noninferiority primary outcome was $a \geq 50 \%$ reduction in apnoea-hypopnoea index (AHI) or achieving $\mathrm{AHI}<10$ events/hour at 2 months. The non-inferiority margin was preset as a difference between groups of $20 \%$ for the primary outcome in the per-protocol analysis.

Main results of 198 patients (mean age 51 [SD, 12] years; 138 [72.6\%] men; mean body mass index 26 [SD, 2.7] kg/m²; mean AHI 26.6/hour [SD, 10.4]), 100 received TALI and 98 ONIRIS. In per-protocol analysis, the response rate was $51.7 \%$ in the TALI group versus $53.6 \%$ in the ONIRIS group (absolute difference 1.9\%; $90 \% \mathrm{Cl}: 11 \%$ to $15 \%$, within the non-inferiority margin). Effectiveness was the same for severity, symptoms, quality of life and blood pressure reduction. Patients in ONIRIS group reported more side effects and adherence was slightly better with TALI.

Conclusion In patients with OSA refusing or not tolerating CPAP, the thermoplastic heat-moulded titratable MAD was non-inferior in the short-term to the custom-made acrylic MAD.

Trial registration number NCT02348970.

\section{INTRODUCTION}

Obstructive sleep apnoea (OSA) is characterised by repetitive episodes of partial or complete pharyngeal obstruction during sleep. ${ }^{1}$ OSA is one of the most frequent chronic diseases with both social and multiorgan consequences making it an economic burden for society. OSA durably impairs the quality of life of patients and their bedpartners and is associated with co-morbidities including hypertension,

\section{Key messages}

What is the key question?

- Is a thermoplastic heat-moulded titratable mandibular advancement device (MAD) noninferior to a custom-made acrylic titratable MAD for the treatment of obstructive sleep apnoea (OSA) in the short term?

What is the bottom line?

- In patients with OSA refusing or not tolerating continuous positive airway pressure, a thermoplastic heat-moulded titratable MAD is non-inferior to a custom-made acrylic MAD at 2 months after implementation.

Why read on?

- This study shows that in patients with moderate-to-severe OSA, a simple, cheap and ready to use thermoplastic heat-moulded titratable MAD can be used to determine whether mandibular advancement therapy is feasible and effective, before ordering a custom-made MAD for long-term use.

arrhythmias, stroke, coronary heart disease and metabolic dysfunction. ${ }^{1}$

Continuous positive airway pressure (CPAP), the first-line therapy for OSA, requires high adherence to be effective in terms of symptom improvement and reduction of the burden of co-morbidities. ${ }^{2}$ Such adherence is difficult to achieve in the long term and mandibular advancement devices (MADs) have emerged as the leading alternative to CPAP. MAD and CPAP are similarly effective on symptoms, quality of life $^{34}$ and in attaining reductions in blood pressure and cardiovascular morbidity. ${ }^{56}$ Although CPAP has a greater effect on apnoea-hypopnoea index (AHI) reduction, adherence is better with MAD explaining the comparable mean disease alleviation achieved by the two treatment modalities. ${ }^{4}$

Despite good tolerance and efficacy, there are still barriers limiting the widespread use of MAD and its acceptance in OSA routine clinical practice. $^{7}$ Various different MAD designs currently exist and constantly emerge on the market without clear evidence regarding the best technical choice and the cost-effectiveness compromise. ${ }^{8}$ Titratable 
two-piece custom-made MADs are considered the gold standard in clinical guidelines but at the price of higher costs and treatment delays for manufacture to customised specifications. Also, the MAD titration (adjustment of the degree of protrusion to optimise therapy) procedures are poorly standardised and the process can last several months with difficulties in predicting long-term effectiveness. Thermoplastic two-piece MADs constructed of a material that becomes mouldable when warmed by immersion in hot water, ${ }^{7}$ recently became titratable and might offer relatively cheap devices for testing efficacy in a given patient and to provide a fast-track treatment pathway before prescription of a more expensive two-piece custom-made MAD for longterm use. ${ }^{9}$ Such a paradigm merits being tested in a randomised controlled head-to-head trial. This study was a pragmatic, multicentre, parallel-group randomised controlled trial to determine whether the two-piece thermoplastic heat-moulded titratable MAD (ONIRIS; ONIRIS SAS, Rueil Malmaison, France) is non-inferior to the two-piece custom-made acrylic titratable MAD (TALI; ONIRIS SAS, Rueil Malmaison, France) in patients with OSA refusing or not tolerating CPAP. The primary outcome was efficacy response at 2 months and secondary outcomes included tolerance and adherence.

\section{MATERIALS AND METHODS \\ Study design and participants}

This was a prospective, multicentre, randomised, controlled, open trial. The study population consisted of adults $(>18$ years) with severe OSA refusing or not tolerating CPAP, without dental, periodontal or temporomandibular joint contraindications ${ }^{1011}$ and naïve to MAD use. In line with the French Respiratory Society consensus, severe OSA was defined as an $\mathrm{AHI} \geq 15$ / hour with either severe daytime sleepiness or at least two of the following symptoms: severe nightly snoring, gasping or choking sensations, unrefreshing sleep, fatigue and/or nocturia. Patients were recruited by private practice sleep clinics and university hospital sleep centres.

The main exclusion criteria were severe psychiatric or neuromuscular disorders (at the investigator's judgement); more than $20 \%$ of central sleep apnoea and hypopnoea; OSA associated with coexistent sleep disorders (narcolepsy, hypersomnia, severe restless legs syndrome); body mass index $>30 \mathrm{~kg} / \mathrm{m}^{2}$; ongoing or scheduled orthodontic treatment; unmanageable gag reflex; pregnant or breastfeeding women; patients with epilepsy; inability to give informed consent; patient included in another ongoing clinical study; and patient not covered by the French health insurance system.

The trial was registered on www.clinicaltrials.gov: NCT02348970 on 28 January 2015. The protocol was approved by the French ethics committee (Comité Protection des Personnes Sud-Ouest et Outre-Mer III, Bordeaux (N: 2014-A01191-46)) and all participants provided written informed consent at the initial visit, 30 days prior to baseline $(D-30)$. The study was done in accordance with Good Clinical Practice guidelines and the Declaration of Helsinki.

\section{Randomisation and masking}

Between 29 January 2015 and 31 December 2016, patients were recruited by sleep specialists in the eight participating centres where an ambulatory respiratory polygraphy (PG) or polysomnography (PSG) was performed during which their AHI was measured. If they fulfilled the inclusion criteria, they were seen by a dentist trained in sleep medicine to assess dental, periodontal or joint contraindications. A visit 15 days prior to
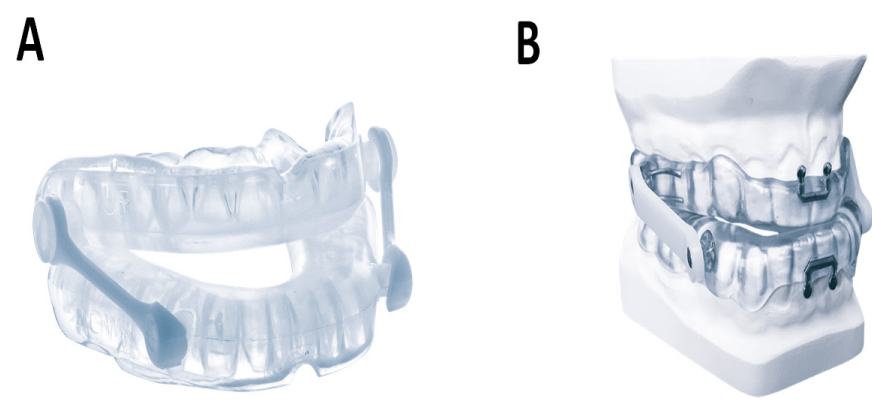

Figure 1 MADs used in the two study arms. (A) ONIRIS is a two-piece innovative adjustable thermoplastic MAD combining a precise teeth impression, is titratable and allows free jaw movements and mouth opening (ONIRIS SAS). (B) TALI is a two-piece acrylic custom-made $M A D$, is titratable and allows free jaw movements and mouth opening (ONIRIS SAS). MADs, mandibular advancement devices.

baseline (D-15) was mainly dedicated to evaluation for MAD contraindications and checking for inclusion-exclusion criteria. In the absence of contraindications, eligible patients were randomly assigned in a 1:1 ratio to one or other of the two treatment groups (custom-made MAD (TALI) or the thermoplastic heat-moulded MAD (ONIRIS)). Stratified randomisation was conducted using a computer-generated allocation sequence accessible from each centre through a secured dedicated website. Stratification was done according to study centres and to initial AHI severity (two levels: $\mathrm{AHI} \leq 30, \mathrm{AHI}>30$ ). Investigators and patients were unmasked to treatment assignment.

\section{Procedures and intervention}

ONIRIS (figure 1) is a two-piece titratable thermoplastic MAD, made of two stiff gutters heat-moulded on plaster-casts of dental arches (or in situ) coupled by two adjustable connecting rods allowing mandibular advancement to be set in steps of $1 \mathrm{~mm}$ and permitting freedom of jaw opening movements. TALI (figure 1) is a two-piece titratable acrylic custom-made MAD allowing one to set the mandibular advancement in steps of $1 \mathrm{~mm}$ and allowing freedom of jaw opening movements. Studies on the efficacy of various MADS have been reviewed by Basyuni et al. ${ }^{7}$

Fifteen days after randomisation, the patient was seen by the dental specialist to fit the MAD. MAD fitting was defined as the study start point (baseline, day 0: D0), when baseline characteristics were collected and patients were asked to complete self-reported questionnaires on snoring intensity (using a visual analogue scale); daytime sleepiness (Epworth Sleepiness Scale); fatigue and depression (Pichot scale); quality of life (ShortForm 12: SF-12) and their resting office blood pressure was measured. Patients were asked to wear their MADs every night while sleeping for the duration of the study. During the study, patients were seen for follow-up visits by the dental specialist at 15,30 and 45 days to perform a MAD titration if needed, and to assess treatment adherence, snoring intensity and blood pressure. At 60 days, patients were seen by the sleep specialist and an ambulatory respiratory PG or PSG was again performed to measure their AHI. The same sleep study methodology was used at diagnosis and when the same patient was wearing a MAD. The proportion of PG versus PSG was not different between groups (43\% of PG vs $57 \%$ of PSG). Full overnight in-laboratory attended PSG or type III cardiopulmonary sleep was used for OSA characterisation. Sleep stages and micro-arousals were scored using the American Academy of Sleep Medicine criteria. ${ }^{12}$ An apnoea was scored if a drop of $90 \%$ or more in airflow signal excursion 
lasting at least $10 \mathrm{~s}$ was observed. Hypopnoea was defined as a drop greater than or equal to $30 \%$ in airflow lasting at least $10 \mathrm{~s}$ and associated with $3 \%$ oxygen desaturation or electroencephalographic arousal. Interpretation of type III recordings was done using hypopnoea criteria (a 30\% drop in airflow and 3\% desaturation). This scoring method has been demonstrated to have the best diagnostic accuracy compared with full PSG. ${ }^{13}$ AHI cut-off values between 15 and $<30$ were used to classify moderate sleep apnoea and a cut-off value $\geq 30$ was considered as severe sleep apnoea. In addition, the following parameters were again measured: snoring intensity (visual analogue scale); Epworth sleepiness scale score; Pichot fatigue and depression scale scores; quality of life (SF-12); compliance to treatment (self-reported); nature, frequency and intensity of adverse events; and office blood pressure.

\section{Outcomes}

The primary efficacy endpoint was treatment response, defined as a decrease in at least $50 \%$ from baseline in the $\mathrm{AHI}$ or an $\mathrm{AHI}$ $<10 /$ hour at the 2-month visit. Secondary efficacy endpoints included changes in AHI, Epworth sleepiness scale score, Pichot fatigue and depression scale scores, snoring intensity measured by the visual analogue scale, health-related quality of life evaluated by the SF-12 form and device adherence. Adherence to treatment was based on patient self-reporting regarding the use of the device and expressed as follows: percentage of nights per week and percentage of hours per night. Adherence results were classified in three classes: poor adherence when MAD was used less than $50 \%$ of the night; good adherence when MAD was used between $50 \%$ and $85 \%$ of the night; and excellent adherence when MAD was used more than $85 \%$ of the night. The rationale was to reflect the adherence thresholds usually accepted for CPAP (ie, 4 hours for $50 \%$ of the night, 4-7 hours for $50 \%-85 \%$ of the night and above 7 hours/night). As for CPAP, we also assessed the percentage of nights wearing MADs. The safety assessment consisted of regular monitoring and recording all adverse events and serious adverse events, which were coded according to MedDRA V.10.8.

\section{Statistical analysis}

The aim of the study was to determine whether a thermoplastic heat-moulded titratable MAD (ONIRIS) was non-inferior to a custom-made acrylic MAD (TALI) in patients with moderate-to-severe OSA refusing or not tolerating CPAP. Based on data retrieved from the literature currently available on MADs, it was estimated that the rate of response at 2 months would be $75 \%$ in both groups and we set a non-inferiority margin of $20 \%$ at 2 months. The non-inferiority margin was defined a priori and was considered as clinically relevant by the panel of investigators.

With a statistical power of $90 \%, 81$ patients needed to be included in each group (Pearson's $\chi^{2}$ test). Taking into account potential loss to follow-up, missing data and dropouts, the study size was increased by $25 \%$. Consequently, a total of 204 patients (102 in each treatment group) were needed to assess the aforementioned hypothesis.

The primary efficacy endpoint was analysed following a non-inferiority hypothesis. The non-inferiority margin was defined as a difference between groups of $20 \%$ for the primary outcome and assessed in per-protocol analysis. According to methodological guidelines on non-inferiority trials, the per-protocol population (PP), which includes all patients who satisfactorily complied with the assigned treatment and who had no major protocol violations, is more likely to identify any treatment differences and was consequently used to perform such an analysis. In the present study, the intention-to-treat population included all randomised patients. The tolerance population (TOL) included all randomised patients who used the device at least once. The PP included all randomised patients who used the device at least once during the 2 months of follow-up with no major deviation regarding the established protocol such as non-conformity to inclusion and exclusion criteria or no assessment of the primary endpoint at the 2-month follow-up visit (except for patients reporting a lack of efficacy in-between visits). An additional sensitivity analysis was performed on the TOL.

Secondary efficacy endpoints were analysed on the TOL using two-tailed tests with an alpha set at 0.05. Intragroup evolutions of secondary efficacy endpoints were analysed using the Cochran-Mantel-Haenszel test and comparisons between groups were performed using $\chi^{2}$ tests.

\section{RESULTS \\ Participants}

Out of the 211 potentially eligible patients, 198 patients were randomised to either the ONIRIS group $(n=98)$ or the TALI group ( $\mathrm{n}=100$ ) (figure 2). The 13 non-randomised patients were excluded for the following reasons: study thought to be too demanding $(\mathrm{n}=1)$; dental, periodontal or joint contraindications $(n=7)$; consent withdrawal $(n=1)$; lost to follow-up between screening and randomisation $(\mathrm{n}=3)$; impossibility to attend follow-up visits $(\mathrm{n}=1)$. Out of the 198 patients with severe OSA refusing or not tolerating CPAP, eight patients (six in the ONIRIS group and two in the TALI group) never used the treatment. Consequently, the TOL included 190 patients. For the per-protocol analysis, 34 patients were excluded from the TOL. Consequently, the PP consisted of 156 patients: 69 in the ONIRIS group and 87 in the TALI group.

\section{Baseline characteristics}

Baseline demographics and clinical characteristics for the TOL and PP (table 1) were well balanced between treatment groups. The included population was typical of moderate-to-severe OSA with one-third of the patients being in the severe AHI range (AHI $>30 / h)$.

\section{Primary outcome (primary analysis in PP)}

According to the predefined criteria of response, 45/87 (51.7\%) and $37 / 69(53.6 \%)$ patients were successfully treated for OSA in the TALI and ONIRIS groups, respectively. The difference does not exceed the non-inferiority margin (difference 0.019 ; $90 \%$ CI: -0.1140 to 0.1516 ) (see figure 3 ). The final mandibular protrusion level was $8 \mathrm{~mm} \pm 2.9$ in the TALI arm versus $8 \mathrm{~mm} \pm 2.1$ in the ONIRIS group.

In the TALI treatment group, out of the 45 patients rated as responders, $28(32.2 \%)$ showed an $\mathrm{AHI}<10 /$ hour (total responders) and $17(19.5 \%)$ showed a decrease in at least 50\% in AHI (partial responders). In the ONIRIS treatment group, out of the 37 patients rated as responders, $28(40.6 \%)$ showed an $\mathrm{AHI}<10 /$ hour and $9(13.0 \%)$ showed a decrease in at least 50\% in $\mathrm{AHI}$.

\section{Primary outcome in the Tolerance population}

To account for the different proportions of dropouts in the two arms, in the TOL population analysis we considered dropouts as failures for the primary outcome. Sensitivity analysis 


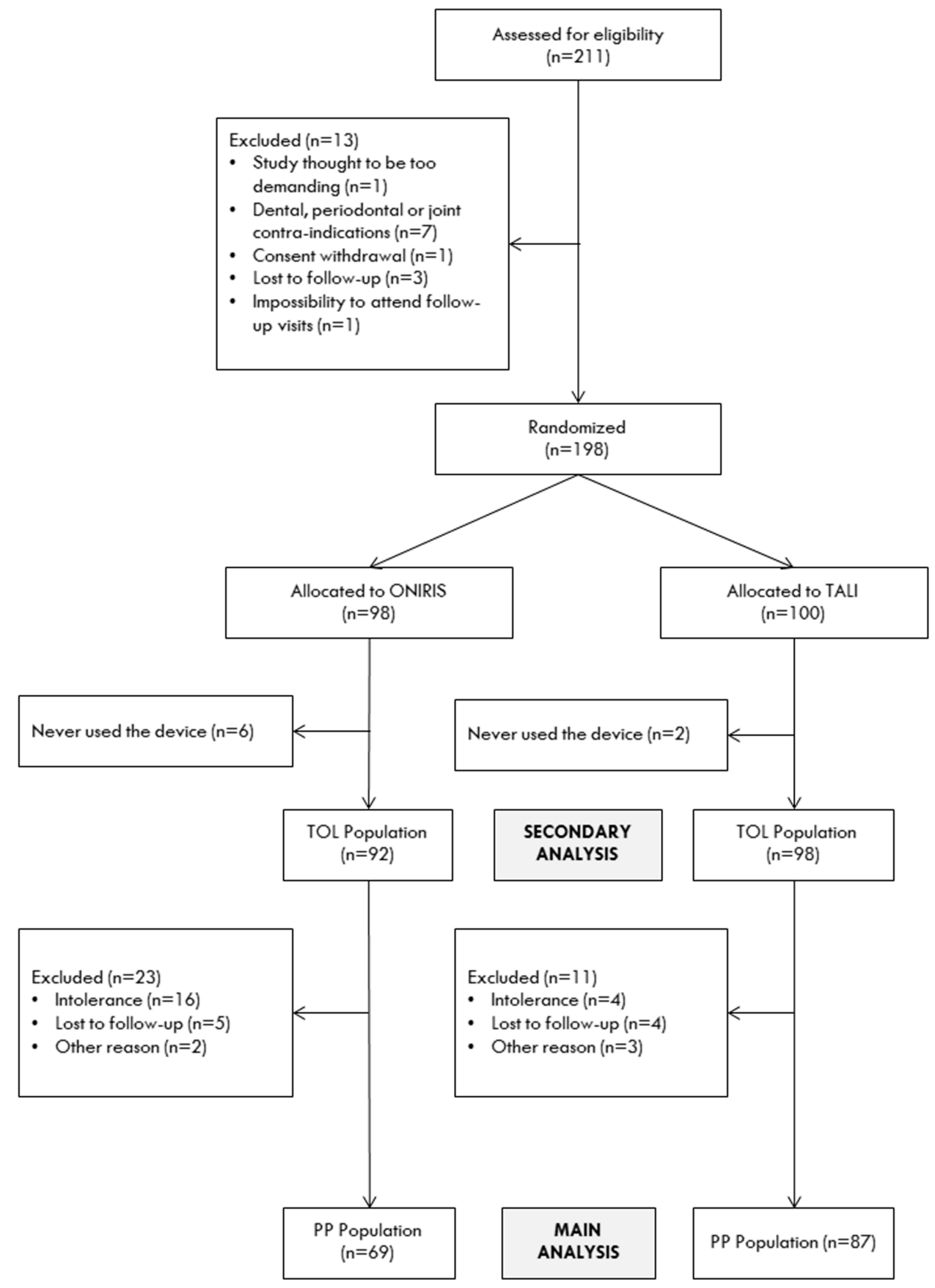

Figure 2 Patient flow-chart. ITT, intention-to-treat population; TOL, tolerance population; PP, per-protocol population.

performed on the TOL population showed that according to the predefined criteria of response, $45.9 \%$ and $40.2 \%$ were successfully treated for OSA in the TALI and ONIRIS groups, respectively, and the difference did not exceed the non-inferiority margin (difference $-0.0517 ; 90 \%$ CI -0.1764 to 0.0631 ) (see figure 3).

\section{Secondary outcomes}

\section{Tolerance population}

After 2 months, both treatments significantly improved AHI per hour, and scores for SF-12 (both the physical and mental subscores), Pichot fatigue and depression scales, Epworth sleepiness scale and snoring (table 2). No significant differences between the thermoplastic MAD (ONIRIS) and the custom-made MAD
(TALI) were observed regarding AHI per hour, SF-12 (physical and mental sections), Pichot depression scales and snoring (see figure 4). A trend but no significant difference in favour of the thermoplastic MAD was observed for the Pichot fatigue scale $(-7.64$ [7.22] versus -5.12 [6.97]; $p=0.0544)$.

At 2 months, self-assessed adherence was rated as good or excellent by $91.4 \%$ of patients in the TALI group (excellent: $82.8 \%$; good: $8.6 \%$ ) and for $74.5 \%$ of patients in the ONIRIS group (excellent: $58.9 \%$; good: $15.6 \%$ ). Overall adherence was significantly better in the TALI group than in the ONIRIS group $(\mathrm{p}=0.0013)$, with reported weekly use (nights/week) and daily use (hours/night) of the devices both significantly better for TALI (6.55 [1.04] vs 5.76 [2.23]; $\mathrm{p}=0.0026$ and 6.6 [1.3] vs 5.2 [2.5]; $\mathrm{p}<0.0001$, respectively). 
Table 1 Baseline demographics and clinical characteristics (tolerance and per-protocol populations)

\begin{tabular}{|c|c|c|c|c|}
\hline & \multicolumn{2}{|c|}{ Tolerance population } & \multicolumn{2}{|c|}{ Per-protocol population } \\
\hline & $\begin{array}{l}\text { TALI } \\
(\mathrm{N}=98)\end{array}$ & $\begin{array}{l}\text { ONIRIS } \\
(\mathrm{N}=92)\end{array}$ & $\begin{array}{l}\text { TALI } \\
(\mathrm{N}=87)\end{array}$ & $\begin{array}{l}\text { ONIRIS } \\
(\mathrm{N}=69)\end{array}$ \\
\hline Age (years) & $\begin{array}{l}52.9(12.3 ; \\
\mathrm{n}=98)\end{array}$ & $\begin{array}{l}49.0(11.60 ; \\
\mathrm{n}=92)\end{array}$ & $\begin{array}{l}52.9(12.2 \text {; } \\
\mathrm{n}=87)\end{array}$ & $\begin{array}{l}49.3(11.2 \text {; } \\
\mathrm{n}=69)\end{array}$ \\
\hline \multicolumn{5}{|l|}{ Gender } \\
\hline - Men & $75(76.5 \%)$ & $63(68.5 \%)$ & $67(77 \%)$ & $50(72.5 \%)$ \\
\hline - Women & $23(23.5 \%)$ & $29(31.5 \%)$ & $20(23 \%)$ & $19(27.5 \%)$ \\
\hline $\begin{array}{l}\text { Body mass index } \\
\left(\mathrm{kg} / \mathrm{m}^{2}\right)\end{array}$ & $\begin{array}{l}26.12(2.80 ； \\
n=98)\end{array}$ & $\begin{array}{l}25.77(2.70 ； \\
\mathrm{n}=91)\end{array}$ & $\begin{array}{l}25.91 \\
\mathrm{n}=87)\end{array}$ & $\begin{array}{l}25.86(2.71 ； \\
\mathrm{n}=68)\end{array}$ \\
\hline \multicolumn{5}{|l|}{ MAD indication } \\
\hline $\begin{array}{l}\text { Initial CPAP } \\
\text { refusal }\end{array}$ & $41(41.8 \%)$ & $39(42.4 \%)$ & $33(37.9 \%)$ & $29(42 \%)$ \\
\hline $\begin{array}{l}\text { Not } \\
\text { tolerating } \\
\text { CPAP }\end{array}$ & $57(58.2 \%)$ & $52(56.5 \%)$ & $54(62.1 \%)$ & $39(56.5 \%)$ \\
\hline AHI per hour & $\begin{array}{l}27.4(10.1 ； \\
\mathrm{n}=98)\end{array}$ & $\begin{array}{l}26.0(10.7 ; \\
\mathrm{n}=92)\end{array}$ & $\begin{array}{l}27.1 \\
n=87)\end{array}$ & $\begin{array}{l}26.1(11.1 ； \\
\mathrm{n}=69)\end{array}$ \\
\hline $\begin{array}{l}\text { Systolic blood } \\
\text { pressure }\end{array}$ & $\begin{array}{l}128.0(19.5 ； \\
\mathrm{n}=97)\end{array}$ & $\begin{array}{l}126.3(14.4 ； \\
\mathrm{n}=92)\end{array}$ & $\begin{array}{l}127.6(19.9 ； \\
\mathrm{n}=87)\end{array}$ & $\begin{array}{l}126.4(13.7 ； \\
\mathrm{n}=69)\end{array}$ \\
\hline $\begin{array}{l}\text { Diastolic blood } \\
\text { pressure }\end{array}$ & $\begin{array}{l}81.0(12.1 ； \\
n=97)\end{array}$ & $\begin{array}{l}81.3(11.3 ; \\
\mathrm{n}=92)\end{array}$ & $\begin{array}{l}81.3(12.3 ; \\
\mathrm{n}=87)\end{array}$ & $\begin{array}{l}81.8(11.4 ; \\
\mathrm{n}=69)\end{array}$ \\
\hline \multicolumn{5}{|l|}{$\begin{array}{l}\text { Hypertension } \\
\text { (WHO criteria) }\end{array}$} \\
\hline - Mild & $20(20.6 \%)$ & $21(22.8 \%)$ & $18(20.7 \%)$ & $15(21.7 \%)$ \\
\hline - Moderate & $7(7.2 \%)$ & $5(5.4 \%)$ & $6(6.9 \%)$ & $4(5.8 \%)$ \\
\hline $\begin{array}{l}\text { No } \\
\text { hypertension }\end{array}$ & $70(72.2 \%)$ & $66(71.7 \%)$ & $63(72.4 \%)$ & $50(72.5 \%)$ \\
\hline $\begin{array}{l}\text { Snoring ( } 10 \mathrm{~cm} \\
\text { on VAS) }\end{array}$ & $\begin{array}{l}6.402(2.259 \text {; } \\
\mathrm{n}=94)\end{array}$ & $\begin{array}{l}6.675(2.371 ； \\
n=86)\end{array}$ & $\begin{array}{l}6.420(2.203 ； \\
\mathrm{n}=84)\end{array}$ & $\begin{array}{l}6.654(2.460 ; \\
n=65)\end{array}$ \\
\hline $\begin{array}{l}\text { Number of } \\
\text { missing teeth }\end{array}$ & $\begin{array}{l}3.9(3.2 ; \\
\mathrm{n}=98)\end{array}$ & $3.4(2.4 ; n=92)$ & $\begin{array}{l}3.8(3.2 ; \\
\mathrm{n}=87)\end{array}$ & $\begin{array}{l}3.4(2.5 ; \\
\mathrm{n}=69)\end{array}$ \\
\hline \multicolumn{5}{|l|}{$\begin{array}{l}\text { Partially } \\
\text { edentulous } \\
\text { patient }\end{array}$} \\
\hline $\begin{array}{l}\text { At least one } \\
\text { missing tooth }\end{array}$ & $83(83.9 \%)$ & 75 (81.2\%) & $73(84.7 \%)$ & $56(81.5 \%)$ \\
\hline $\begin{array}{l}\text { At least four } \\
\text { missing teeth }\end{array}$ & $56(57.1 \%)$ & $50(54.4 \%)$ & $49(56.3 \%)$ & $37(53.6 \%)$ \\
\hline
\end{tabular}

Data are $\mathrm{n}(\%)$ or mean (SD; number of patients), unless otherwise indicated. The tolerance population was defined as all randomised patients who used the device at least once. The per-protocol population was defined as all randomised patients who used the device at least once with no major deviation regarding the established protocol such as non-conformity to major inclusion and exclusion criteria, and no assessment of the primary endpoint at 2-month follow-up visit (except for patients reporting a lack of efficacy in-between visits).

AHI, apnoea-hypopnoea index; CPAP, continuous positive airway pressure; MAD,

Mandibular advancement device; VAS, visual analogue scale.

\section{Per-protocol population}

Significant improvements from baseline were also observed for secondary outcomes in the PP in both groups. A trend, but not significant difference, in favour of the thermoplastic MAD was observed for the Epworth Sleepiness score (-4.43 [3.87] vs -3.48 [3.82]; $p=0.0701$ ).

When considering the PP, self-assessed adherence was rated as good or excellent for $94 \%$ of patients in the TALI group (excellent: $86.9 \%$; good: $7.1 \%$ ) and for $89.7 \%$ of patients in the ONIRIS group (excellent: 69.1\%; good: 20.6\%). Overall

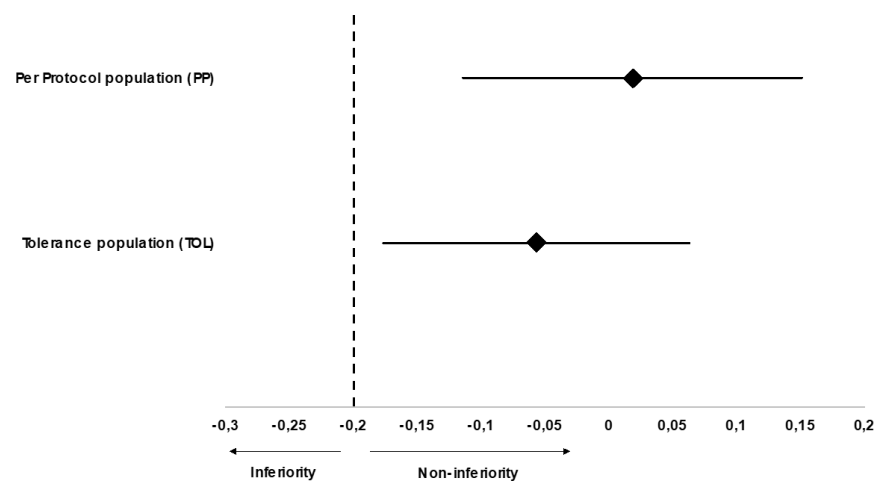

Figure 3 Responder rate difference. The difference in response rates in both groups for per-protocol and tolerance populations is expressed as the mean (diamonds). The non-inferiority margin with a $90 \% \mathrm{Cl}$ was preset at -0.2 (dashed line). Diamonds represent the mean difference between the two treatment groups and lines represent the $90 \% \mathrm{Cl}$. Whatever the studied population, the difference did not exceed the noninferiority margin.

adherence was still significantly better in the TALI group $(\mathrm{p}=0.0228)$, as well as nightly use (6.8 [1.1] vs 6.1 [1.5]; $\mathrm{p}=0.044)$. However, no significant difference in weekly use (number of nights/week) was observed between the groups $(6.64$ [0.98] vs 6.43 [1.46]; $\mathrm{p}=0.2971)$.

\section{Adverse events}

At 2 months, no serious adverse event was reported in either treatment group. During the first 2 months, $77.6 \%$ of patients with TALI and $87.0 \%$ of patients with ONIRIS reported discomfort at least once $(p=0.091)$. The most frequently reported side effects were dental pain (16.8\%), temporomandibular joint pain (14.7\%), discomfort related to MAD volume in the mouth (11.6\%), muscular pain (10.5\%) and muscle ache (10.0\%).

In the first 2 weeks, significantly more muscle aches were reported in the TALI group $(14.3 \%$ vs $5.4 \%, \mathrm{p}=0.0421)$. Whereas significantly more discomfort due to the encumbrance of the device in the mouth $(19.6 \%$ vs $4.1 \%, \mathrm{p}<0.001)$, excessive salivation $(14.1 \%$ vs $2.0 \%, \mathrm{p}=0.002)$ and gag reflex $(5.4 \%$ vs $0 \%, p=0.0193)$ were observed in the ONIRIS group.

After 2 months, the number of patients reporting discomfort dramatically decreased: by $16.3 \%$ in the TALI group and $21.8 \%$ in the ONIRIS group. No significant differences between groups were observed $(p=0.166)$. Among patients reporting discomfort only one (1\%) in the TALI group and three (3.3\%) in the ONIRIS group reported discomfort that overshadowed treatment benefits.

\section{DISCUSSION}

This is the first randomised controlled trial providing a headto-head comparison between a two-piece thermoplastic heat-moulded titratable MAD (ONIRIS) and a two-piece custom-made acrylic reference titratable MAD (TALI) in patients with severe OSA refusing or intolerant to CPAP. Evaluation at 2 months demonstrated clear non-inferiority of the thermoplastic heat-moulded titratable MAD (ONIRIS). The non-inferiority was true not only for the primary outcome (rate of response) but also for parameters of OSA severity (AHI), patient-centred outcomes including symptoms and quality of life, and co-morbidities (blood pressure reduction). The burden of side effects was higher with the thermoplastic MAD but this was essentially during the first couple of weeks of treatment initiation and the 
Table 2 Changes in measurements from baseline to end of intervention for secondary outcomes at 2 months (tolerance population)

\begin{tabular}{|c|c|c|c|}
\hline & TALI & ONIRIS & $p$ value \\
\hline AHI per hour & $\begin{array}{l}-11.16(10.80 ; \\
\mathrm{n}=87)^{\star * *} \\
\text { IQR }:-19.00 \text { to } \\
-5.00 \\
\text { Median: }-11.00\end{array}$ & $\begin{array}{l}-11.90(9.43 ; \\
\mathrm{n}=69)^{* * *} \\
\text { IQR }:-17.00 \text { to } \\
-7.00 \\
\text { Median: }-10.00\end{array}$ & $0.4025(t)$ \\
\hline Snoring (VAS) & $\begin{array}{l}-3.70(2.88 ; \\
\mathrm{n}=94)^{*} \\
\text { IQR }:-6.00 \text { to } \\
-1.45 \\
\text { Median: }-4.00\end{array}$ & $\begin{array}{l}-4.03(2.88 ; \\
\mathrm{n}=86)^{*} \\
\text { IQR : }-6.65 \text { to } \\
-1.30 \\
\text { Median: }-3.60\end{array}$ & $0.4504(t)$ \\
\hline Epworth sleepiness & $\begin{array}{l}-3.34(3.77 ; \\
\mathrm{n}=95)^{* * *} \\
\text { IQR }:-6.00 \text { to } \\
-1.00 \\
\text { Median: }-3.00\end{array}$ & $\begin{array}{l}-3.76(4.16 ; \\
\mathrm{n}=87)^{* * *} \\
\text { IQR }:-7.00 \text { to } \\
-2.00 \\
\text { Median: }-3.00\end{array}$ & $0.2143(t)$ \\
\hline Pichot fatigue & $\begin{array}{l}-5.12(6.97 ; \\
\mathrm{n}=81)^{\star * *} \\
\text { IQR }:-9.00 \text { to } \\
-1.00 \\
\text { Median: }-4.00\end{array}$ & $\begin{array}{l}-7.52(7.22 ; \\
\mathrm{n}=60)^{* * *} \\
\text { IQR : }-14.00 \text { to } \\
-2.00 \\
\text { Median: }-6.00\end{array}$ & $0.0544(t)$ \\
\hline Pichot depression & $\begin{array}{l}-1.44(3.23 ; \\
\mathrm{n}=79)^{* *} \\
\text { IQR : }-3.00 \text { to } 0.00 \\
\text { Median: }-1.00\end{array}$ & $\begin{array}{l}-2.12(3.31 \\
\mathrm{n}=59)^{* * *} \\
\text { IQR : }-4.00 \text { to } 0.00 \\
\text { Median: }-1.00\end{array}$ & $0.2240(t)$ \\
\hline SF-12 Mental score & $\begin{array}{l}5.27(17.68 ; \\
\mathrm{n}=81)^{* *} \\
\text { IQR }:-7.91 \text { to } \\
18.31 \\
\text { Median: } 6.59\end{array}$ & $\begin{array}{l}9.07(21.25 ; \\
\mathrm{n}=60)^{* * *} \\
\text { IQR : }-4.0 \text { to } 22.57 \\
\text { Median: } 8.39\end{array}$ & $0.4441(t)$ \\
\hline SF-12 physical score & $\begin{array}{l}4.22(14.81 ; \\
\mathrm{n}=81)^{*} \\
\text { IQR : }-6.23 \text { to } \\
12.10 \\
\text { Median: } 5.21\end{array}$ & $\begin{array}{l}7.71(13.02 ; \\
\mathrm{n}=60)^{* * *} \\
\text { IQR : } 0.72 \text { to } 14.55 \\
\text { Median: } 5.96\end{array}$ & $0.2967(t)$ \\
\hline $\begin{array}{l}\text { Diastolic blood } \\
\text { pressure } \\
\text { (patients with } \\
\text { hypertension at } \\
\text { inclusion) }\end{array}$ & $\begin{array}{l}-7.04(10.85 ; \\
\mathrm{n}=26)^{* *} \\
\text { IQR }:-13.00 \text { to } \\
-4.00 \\
\text { Median: }-7.50\end{array}$ & $\begin{array}{l}-4.84(12.80 ; \\
\mathrm{n}=25) \\
\text { IQR : }-10.00 \text { to } \\
5.00 \\
\text { Median: }-5.00\end{array}$ & $0.3796(W)$ \\
\hline $\begin{array}{l}\text { Systolic blood pressure } \\
\text { (patients with } \\
\text { hypertension at } \\
\text { inclusion) }\end{array}$ & $\begin{array}{l}-11.19(16.07 ; \\
\mathrm{n}=26)^{* * *} \\
\text { IQR }:-17.00 \text { to } \\
-5.00 \\
\text { Median: }-9.00\end{array}$ & $\begin{array}{l}-4.36(17.42 ; \\
\mathrm{n}=17) \\
\text { IQR : }-18.00 \text { to } \\
5.00 \\
\text { Median: }-4.00\end{array}$ & $0.2091(W)$ \\
\hline
\end{tabular}

Data are mean (SD; number of patients) and median [IQR]. (†) ANCOVA; (W)

Wilcoxon test; significance of evolution from baseline: ${ }^{*} p<0.05 ;{ }^{* *} p<0.01$;

$* * * p<0.0001$ (paired t-test).

AHI, apnoea-hypopnoae index; ANCOVA, analysis of covariance; Snoring, Epworth and pressure (systolic and diastolic) were analysed using the Last Observation Carried Forward method;VAS, visual analogue scale.

difference disappeared over time. MAD adherence was slightly higher with the acrylic reference MAD but although statistically significant the difference was not clinically relevant.

CPAP and MADs are now considered as being nearly equally effective for treating moderate-to-severe sleep apnoea. ${ }^{3514}$ The main problem for more widely disseminating the prescription of MADs by routine sleep clinics is the huge heterogeneity in the design and complexity of the different devices available on the market. There is consensus in the sleep community towards the abandonment of prefabricated, non-adjustable, over-the-counter 'boil and bite' appliances that are associated with lower rates of efficacy, fall out more easily during the night and suffer from poor tolerance and lower adherence. ${ }^{15-18}$ On the other end of the sophistication spectrum, two-piece custom-made, titratable MADs built by a qualified dentist still appear to be the gold standard according to clinical guidelines. ${ }^{18}$ However, these MADs require several weeks to be manufactured, are more expensive and their efficacy is still difficult to predict. Experiencing failure of these high cost MADs even after titration might generate patient frustration and loss of cooperation for alternative treatments. Also, the degree of MAD sophistication influences cost-effectiveness ratios $^{17}$; and finally, there is limited evidence as to which type of MAD is the best compromise in the treatment of mild-to-moderate OSA. Our study evaluated the thermoplastic titratable ONIRIS MAD fitted by a qualified dentist which was immediately available to the patients. It is cheaper and allows treatment delays to be reduced compared with classic custom-made devices. In a bicentric cohort, Gagnadoux et al evaluated the efficacy of another titratable, thermoplastic MAD compared with a custom-made MAD. ${ }^{9}$ They found no efficacy differences either during PSG or in clinical outcomes. However, the Gagnadoux study ${ }^{9}$ was flawed by the usual limitations of observational studies while the robustness of our data is supported by a head-to-head comparison in a randomised controlled trial design. We clearly established that in patients with OSA refusing or not tolerating CPAP, the thermoplastic heat-moulded titratable MAD was non-inferior to the custommade acrylic MAD in terms of the rate of response, reduction in indices of OSA severity, patient-centred outcomes and blood pressure improvement.

Side effects were clearly more frequent in the first couple of weeks of treatment with the thermoplastic MAD compared with the reference one. This is essentially explained by differences in mouth encumbrance between the two appliances. Interestingly, over time, differences in reported side effects disappeared between the two arms. Moreover, reported adherence was high and above 6 hours per night in both arms despite more discomfort with the thermoplastic MAD. There was a slight statistical difference in favour of the acrylic MAD but without clinical relevance as demonstrated by the same improvements in quality of life and objective outcomes such as blood pressure, in the two arms.

Regarding overall efficacy of the two devices, the definition of response to MADs used in the current study is typical of thresholds previously employed across published studies. ${ }^{3}$ In fact, response rates were $40 \%$ in the TOL population because dropouts were included and considered as failures and non-responders. In the reference per protocol population, the response rate was above $50 \%$ for the two devices and the mean $\mathrm{AHI}$ reduction ( -11 per hour) was in exactly the same range as has been previously reported in randomised controlled trials comparing MADs vers conservative treatment. ${ }^{3}$

\section{Strengths and limitations}

Our study is one of the largest randomised controlled trials in the field of MAD and was adequately powered to address a question relevant to routine OSA clinical practice. This was a multicentre trial including both academic and private practice centres. This suggests that our data are generalisable across different sleep practices and services. The main outcome was at the 2-month visit, but patients were asked to wear the device for 1 year with outcomes, including tolerability, assessed at 12 months (ongoing data analysis not presented here). The expected lifespan of the thermoplastic device might vary between patients and is clearly 


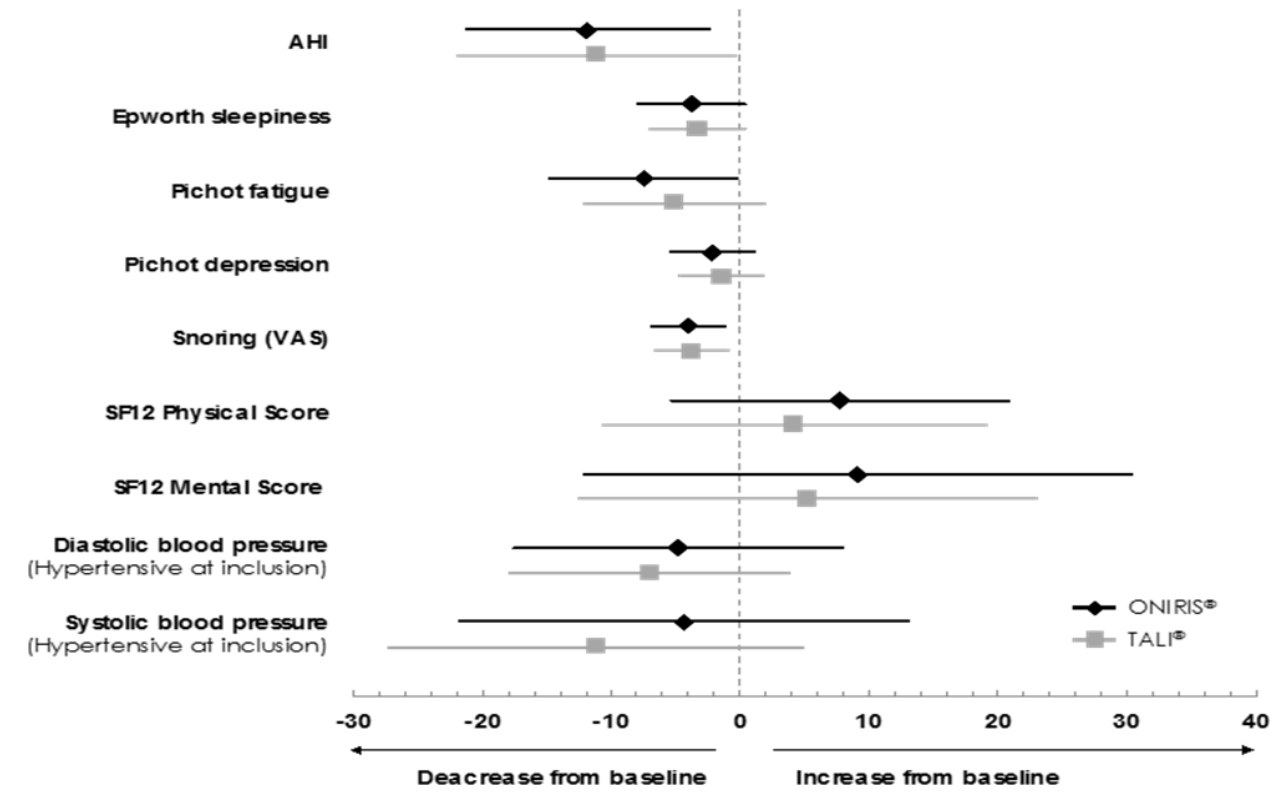

Figure 4 Secondary outcomes: changes from baseline per treatment group (tolerance population). Diamonds and squares represent the mean change from baseline to 2 months in the ONIRIS and TALI groups, respectively. Segments represent the SD. Snoring, Epworth sleepiness and blood pressure (systolic and diastolic) were analysed using the last observation carried forward method. AHI, apnoea-hypopnoea index; VAS, visual analogue scale.

linked to local hygiene. The mean expected lifespan is 2 years. The rate of success with MAD differed between centres but the non-inferiority was confirmed across centres. The parallel-group design did not allow an assessment of whether responses to both devices would be equivalent in the same patients. Our results are certainly specific to the studied MADs but most importantly suggest that the optimisation of thermoplastic devices including the capability of being titratable will constitute a valuable treatment option. Missing teeth affects the efficacy and tolerability to MAD. In contrast to previous studies, we did not exclude patients with missing teeth; our patients had a mean of about four missing teeth (table 1). This increases the relevance of our study to the often middle-aged or elderly OSA population. The main limitation of our study was the absence of a cost-effectiveness assessment that merits further study.

\section{CONCLUSION}

Our quality pragmatic randomised controlled trial demonstrated that in patients with OSA refusing or not tolerating CPAP, a thermoplastic heat-moulded titratable MAD was non-inferior to a custom-made acrylic MAD at 2 months. Such a thermoplastic heat-moulded titratable MAD might represent a simple, cheaper and clinically feasible method of identifying patients likely to benefit from long-term MAD therapy before prescription of a more costly device.

\footnotetext{
Author affiliations

${ }^{1}$ HP2 (Hypoxia Pathopysiologies) Laboratory, Universite Grenoble Alpes, Saint-Martind'Heres 38400, France

${ }^{2}$ Rééducation et Physiologie, Pôle Locomoteur, Grenoble Alpes University Hospital (CHU), Grenoble, France

${ }^{3}$ Sleep Apnoea Centre, New Bel-Air Clinic, Bordeaux, France

${ }^{4}$ Perpignan Sleep Medicine Practice, Perpignan, France

5 Les Pavillons sous Bois ENT Practice, Les Pavillons sous Bois, France

${ }^{6}$ Ear, Nose and Throat Department, New Bel-Air Clinic, Bordeaux, France

${ }^{7}$ Odontology Department, Hôpital Bretonneau, AP-HP, Paris, French Polynesia

${ }^{8}$ Department of Pneumology, CHRU Montpellier, Montpellier, France

${ }^{9}$ HP2 (Hypoxia Pathopysiologies) Laboratory, INSERM U1042 Unit, University

Grenoble Alpes, Grenoble, France
}

${ }^{10}$ EFCR (cardio-respiratory function explorations) Laboratory, Pole Thorax and Vessels, Grenoble Alpes University Hospital (CHU), Grenoble, France

${ }^{11}$ Medical Information, CHRU Montpellier, UMR 729 MISTEA, University of Montpellier I, Montpellier, France

Acknowledgements We thank Dr Jean-Pierre Mallet for the inclusion of patients. Medical writing support was provided by Adrien Pasquier, an independent medical writer. The manuscript was critically edited by Dr Alison Foote (Grenoble Alpes University Hospital).

Contributors JLP reviewed the literature, designed the study, interpreted the results, wrote and revised the article and approved the submitted version. NR reviewed the literature, designed the study, included patients and collected data, interpreted the results, revised the manuscript and approved the submitted version. $\mathrm{OL}$ included patients and collected data, participated in interpreting the results, revised the manuscript and approved the submitted version. $A B$ included patients and collected data, participated in interpreting the results, revised the manuscript and approved the submitted version. JF included patients and collected data, participated in interpreting the results, revised the manuscript and approved the submitted version. EB included patients and collected data, participated in interpreting the results, revised the manuscript and approved the submitted version. $A B$ included patients and collected data, participated in interpreting the results, revised the manuscript and approved the submitted version. SL included patients and collected data, participated in interpreting the results, revised the manuscript and approved the submitted version. RT reviewed the literature, designed the study, included patients and collected data, interpreted the results, revised the manuscript and approved the submitted version. NM reviewed the literature, designed the study, included patients and collected data, interpreted the results, critically revised the article and approved the submitted version.

Funding This study was funded by ONIRIS (France). Data collection, quality control, management and analysis of the data were performed by the contract research organisation Euraxi (France). This work was also supported by the French National Research Agency (Agence Nationale de la Recherche) in the framework of the 'Investissements d'avenir' program (ANR-15-IDEX-02). The funding sources had no role in the study design, realisation, analyses, data interpretation, in writing the manuscript or in the decision to submit it for publication.

Competing interests Drs. Pépin and Raymond received honoraria from ONIRIS for participation in the steering committee, participation in the study and travel expenses to present the results at meetings; Dr Molinari received honoraria for participation in the steering committee and participation in the study; Dr Forcioli received travel expenses to present the results at meetings. Investigators' institutions (for JLP, NR, OL, NA, JF, EB, AB, SL, RT and NM) were remunerated for study visits by ONIRIS. 
Patient consent for publication Not Required

Provenance and peer review Not commissioned; externally peer reviewed.

Data sharing statement De-identified participant data on baseline characteristics and study outcomes will be shared for justified clinical research purposes only, on written request to the study sponsor: Mr Thibault Vincent ONIRIS France (thibault. vincent@oniris-ronflement.fr).The data will be available after publication of the article describing and analysing it. Access will be for clinical researchers affiliated to a recognised university, clinical research centre or hospital, for the purpose of meta-analyses, etc.

Executive steering committee Nathalie Raymond (Principal Investigator), Jean Louis Pépin and Nicolas Molinari (Scientific Advisors). The scientific Steering Committee designed the study and was responsible for its clinical and scientific conduct, and publication of the results. The Steering Committee and in particular first and last authors had full access to all of the data and take responsibility for the integrity and accuracy of the data analysis.

\section{REFERENCES}

1 Lévy P, Kohler M, McNicholas WT, et al. Obstructive sleep apnoea syndrome. Nat Rev Dis Primer 2015;1.

2 Drager LF, McEvoy RD, Barbe F, et al. Sleep apnea and cardiovascular disease: lessons from recent trials and need for team science. Circulation 2017;136:1840-50.

3 Sharples LD, Clutterbuck-James AL, Glover MJ, et al. Meta-analysis of randomised controlled trials of oral mandibular advancement devices and continuous positive airway pressure for obstructive sleep apnoea-hypopnoea. Sleep Med Rev 2016;27:108-24.

4 Hamoda MM, Kohzuka Y, Almeida FR. Oral Appliances for the management of OSA: an updated review of the literature. Chest 2018;153:544-53.

5 Bratton DJ, Gaisl T, Wons AM, et al. CPAP vs mandibular advancement devices and blood pressure in patients with obstructive sleep apnea: a systematic review and meta-analysis. JAMA 2015;314:2280-93.

6 Anandam A, Patil M, Akinnusi M, et al. Cardiovascular mortality in obstructive sleep apnoea treated with continuous positive airway pressure or oral appliance: an observational study. Respirology 2013;18:1184-90.

7 Basyuni S, Barabas M, Quinnell T. An update on mandibular advancement devices for the treatment of obstructive sleep apnoea hypopnoea syndrome. J Thorac Dis 2018;10(Suppl 1):S48-S56.
8 Sharples L, Glover M, Clutterbuck-James A, et al. Clinical effectiveness and cost-effectiveness results from the randomised controlled trial of oral mandibular advancement devices for obstructive sleep apnoea-hypopnoea (TOMADO) and long-term economic analysis of oral devices and continuous positive airway pressure. Health Technol Assess 2014;18:1-296.

9 Gagnadoux F, Nguyen X-L, Le Vaillant M, et al. Comparison of titrable thermoplastic versus custom-made mandibular advancement device for the treatment of obstructive sleep apnoea. Respir Med 2017;131:35-42.

10 Petit F-X, Pépin J-L, Bettega G, et al. Mandibular advancement devices: rate of contraindications in 100 consecutive obstructive sleep apnea patients. Am J Respir Crit Care Med 2002;166:274-8.

11 Gagnadoux F, Pépin J-L, Vielle B, et al. Impact of mandibular advancement therapy on endothelial function in severe obstructive sleep apnea. Am J Respir Crit Care Med 2017; 195:1244-52.

12 Berry RB, Budhiraja R, Gottlieb DJ, et al. Rules for scoring respiratory events in sleep: update of the 2007 AASM manual for the scoring of sleep and associated events. deliberations of the sleep apnea definitions Task Force of the American Academy of sleep medicine. J Clin Sleep Med 2012;8:597-619.

13 Vat S, Haba-Rubio J, Tafti M, et al. Scoring criteria for portable monitor recordings: a comparison of four hypopnoea definitions in a population-based cohort. Thorax 2015;70:1047-53.

14 Bratton DJ, Gaisl T, Schlatzer C, et al. Comparison of the effects of continuous positive airway pressure and mandibular advancement devices on sleepiness in patients with obstructive sleep apnoea: a network meta-analysis. Lancet Respir Med 2015;3:869-78.

15 Johal A, Haria P, Manek S, et al. Ready-Made versus custom-made mandibular repositioning devices in sleep apnea: a randomized clinical trial. J Clin Sleep Med 2017;13:175-82

16 Johal A, Agha B. Ready-made versus custom-made mandibular advancement appliances in obstructive sleep apnea: a systematic review and meta-analysis. J Sleep Res 2018;27:e12660.

17 Quinnell TG, Bennett M, Jordan J, et al. A crossover randomised controlled trial of oral mandibular advancement devices for obstructive sleep apnoea-hypopnoea (TOMADO). Thorax 2014;69:938-45.

18 Ramar K, Dort LC, Katz SG, et al. Clinical practice guideline for the treatment of obstructive sleep apnea and snoring with oral appliance therapy: an update for 2015. J Clin Sleep Med JCSM Off Publ Am Acad Sleep Med 2015;11:773-827. 